\title{
Warp knitted seamless garment CAD technology aided design
}

\author{
Xiaomei Zhang ${ }^{1, \text { a }}$ \\ ${ }^{1}$ Jiangxi Institute of Fashion Technology, Jiangxi, Nanchang, 330201 \\ a775245285@qq,com
}

\begin{abstract}
Keywords: Warp knitted seamless garment; Computer aided design; CAD technology
\end{abstract}
\begin{abstract}
With the rapid development of science and technology, the application of the computer is more and more widely, not only brought great convenience to the life, at the same time also prompted some emerging things arises at the historic moment, such as: warp knitted seamless garment, it is the use of computer-aided design CAD for the design and development, compared with the traditional clothing, not only save the design time, and more simplify the production process, is of great significance to the development of clothing industry, this article mainly from the system set up, production technology and principle to discusses the computer aided design of warp knitted seamless garments.
\end{abstract}

\section{Introduction}

With the rapid development of science and technology, the application of the computer is more and more widely, not only brought great convenience to the life, at the same time also prompted some emerging things arises at the historic moment, such as: warp knitted seamless garment, it is the use of computer-aided design CAD for the design and development, compared with the traditional clothing, not only save the design time, and more simplify the production process, is of great significance to the development of clothing industry, this article mainly from the system set up, production technology and principle to discusses the computer aided design of warp knitted seamless garments.

\section{Warp knitted seamless production technology development condition and the forming principle}

Seamless knitting is based on the traditional knitting industry, the use of computer processing machine to weave a new industry. It is divided into three classes of warp knitting, weft, flat knitting, at present, the warp knitted seamless garment system development and equipment relative to other two kind of more mature, especially in the filar socks, underwear, jumpsuits etc on the production of the weft, flat knitting on technologies and systems development, equipment remains to be studied.

Modern warp knitted seamless production technology is the use of full computer jacquard raschel warp knitting machine, double needle bed by configuring the two parallel columns and back to back to make the production of warp knitting needle bed forming seamless garment. Its production process is composed of four door technology, namely (1) and equipment technology, the production and processing machinery, in the $1980 \mathrm{~s}$, the first mechanical warp knitting machine was born in a foreign country, through constant research and development, Japan in 2001 to study and promote the use of the full computer jacquard raschel double needle bed warp knitting machine. On the equipment of our country although has a gap with foreign level, but also developed RDJ, GE, ZMY series of full computer jacquard raschel warp knitting machine, double needle bed machine speed can reach 500 RPM, immediately can reach 28 needle. (2) technology, in terms of academic, since 1988 in China, some scholars of the hall of warp knitting seamless garment technology in-depth research, either from the perspective of the process principle of clothing or clothing style design, comfort, etc., have made certain achievements, is mainly engaged in seamless machines and abroad of new products, promote the use and reported few studies of its technology. (3) to MES 
technology refers to the production information management system, namely through the MES and seamlessly connected machine control system, to make information timely feedback to the workshop production management system, so that real-time monitoring record production, to timely solve the problems of production. (4) CAD technology, is the core of warp knitting seamless garment industry to modernize, not only from the design of the clothing CAD technology, in the process of implementation, data output all fall within the scope of CAD technology, not only saves production time from the cost control, and on the quality of the product itself to improve the precision of design, make the enterprise get considerable economic benefit.

Warp knitted seamless garment the forming principle of a higher forming and reverse forming can be divided into two kinds, synthetic forming refers to the front and back of warp knitting machine needle bed configuration a full number (jia card stripping comb, using the full number of jacquard stripping comb features, its need two transverse organization control direction, on the other hand, the inverse kinematics is called reverse forming.

\section{Warp knitted seamless garment CAD technology aided design prototype modeling}

Irregularity and complexity of $3 \mathrm{~d}$ mesh will directly affect the treatment effect and efficiency of design system, the rules to the convex hull of the profile of $3 \mathrm{~d}$ model sample make rules of irregular triangular meshes into triangular mesh, and then make the $3 \mathrm{~d}$ model through computer programming into the two-dimensional prototype of warp knitted seamless garment, this is the computer aided design of two-dimensional templates, has an important basic role for garment CAD design.

Coat prototype modeling. Coat prototype modeling are divided into three steps: first, to extract human body feature points, including waist circumference, chest circumference points, axillary points, shoulder peak point, neck, etc., through based on the human body model prototype, according to its characteristic points and the new human body contour line according to the proportional spacing on the $\mathrm{Y}$ axis what determine the feature points in the contour line first, second, the calculation of the new characteristics of the contour point with the original feature point displacement, choose the minimum value of contour vertices is the new feature points, notes for $\mathrm{T}=$ $\{I, j\}$, I as the contour line, $\mathrm{j}$ for the contour vertices minimum value relative to the original feature points of the $\mathrm{Y}$ axis displacement. Second, correlation dimension calculation, warp knitted seamless coat clothing is coat formed on the basis of the prototype structure are measured.2d pattern, the size of the jacket need the parameters of the prototype structure, basic dimensions including the chest, back, shoulder width, neck line, long sleeve arc length, chest width, etc., before and after the half as well as determine the axillary circumference before and after as shown in figure $1 \mathrm{C}$ and $\mathrm{D}$. Among them, the $\mathrm{C}$ point is human body surface axillary the top point, point $\mathrm{D}$ is human body surface axillary lowest point.

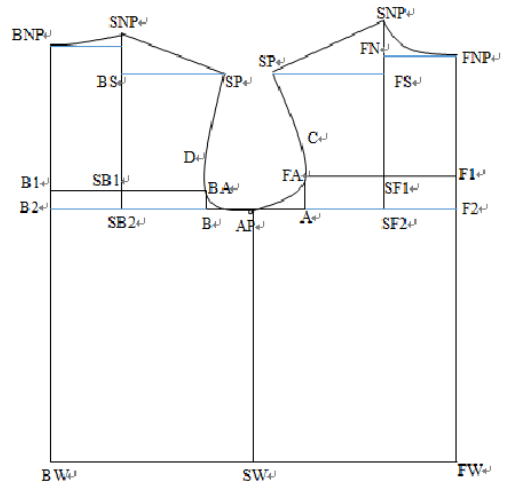

Fig. 1 The paper prototype structure

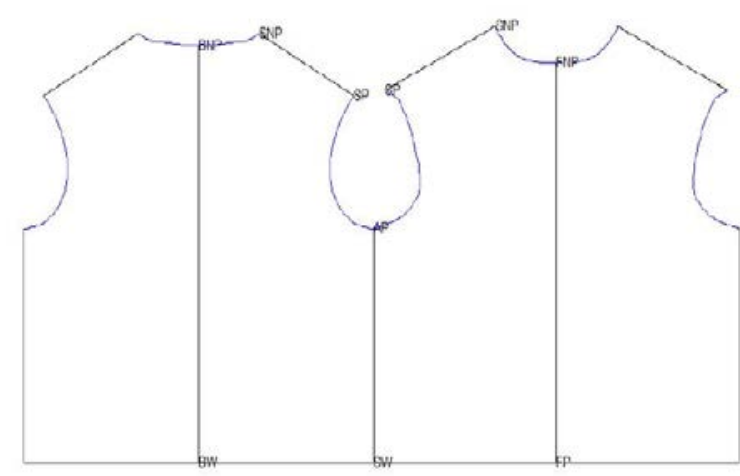

Fig.2Two-dimensional prototype pieces

Third, prototype model, as shown in figure 1, according to the prototype model to calculate the above points to form line generation of warp knitting seamless coat prototype two-dimensional model, the steps are as follows: (1) the length of the computing formula one - FNP pieces (2) F1 as 
A 2 d coordinate origin (3) o FNP, namely the FI - FNP length on the y axis of FN (4), namely do FNP parallel lines, launches the SF1 FNP - length of FN (5), namely the FN do with $x$ axis and y axis parallel lines l FS, SNPS point 6 o, in l, by expanding the FN - length of SNP SNP point, launches the FN - FS length for the FS point 7 on the basis of l, FN for the endpoint was the FN FNP and FN - length of SNP, FN - SNP on each vertex as A parameter, and FN - FNP vertex coordinates pet-name ruby by SF1 - FA and SF1 - the length of the FS, FA - SP on each vertex as A parameter, o FA - SP vertex coordinates attending FA as the endpoint, the length of the FA - A out to $\mathrm{y}$ and allowed to point A loose quantity obtained (11) endpoint for A, A - the length of the AP to the $\mathrm{x}$ axis, and the AP (12) A - AP and A - FA length, FA - AP on each vertex as A parameter, o FA - 13 vertex coordinates of the AP to AP for the endpoint, AP - the length of the SW to the y axis, and the SW (14) SW do $x$ axis parallel lines/y in FW 15) based on bezier curve fitting FNP - SNP vertex, and the curve of FNP - SNP (16) similarly on the steps, fitting AP - SP vertex, and the curve of the AP SP. As shown in figure 2, that is, get the two-dimensional prototype of warp knitted seamless jacket.

Skirt bottoms prototype modeling. Skirt bottoms prototype relative to coat prototype is simple, basic are the different directions of the human body from the waist down as sample vertical formation of the straight down type, straight type belongs to the cylinder structure, its three-dimensional slow start is a rectangle, the length of the skirt of degrees, and the width to the length of skirt. Just make sure the skirt surrounded degree, you can use the algorithm of coat, bottoms contour model is established to capture the human body model, the rules of generating triangular mesh, and the final conversion generated prototype model of warp knitting seamless bottoms. By intercepting plane of the body and bottoms surrounded degree size table waist circumference and hip circumference, and Alpha - Shape algorithm to calculate its convex hull as bottoms surrounded degree size. Waist circumference, hip circumference contour extraction is shown in figure 3, in the system intercepting three-dimensional human body model of lower limb, for establishing $3 \mathrm{~d}$ convex hull body skirt contour model, as shown in figure 4.

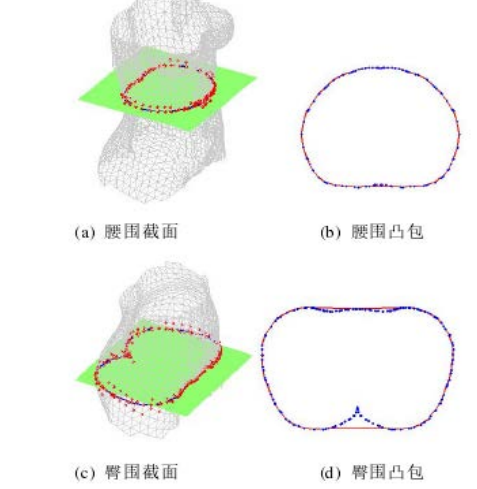

Fig.3 Waist circumference, hip circumference contour extraction
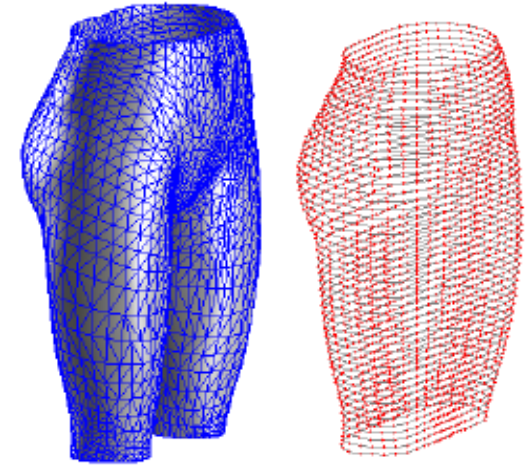

Fig.4 Dress contour model

\section{Warp knitted seamless garment CAD technology design system}

Computer aided design, that is, using CAD technology to design, to reform the traditional clothing design has a central role, it is the use of CAD and graphics machine to clothing style, pattern and so on to carry on the design process. Warp knitted seamless garment computer design system is mainly divided into four aspects.

First, on the system structure, main realization of three-dimensional human body and clothing import and export, apply colours to a drawing, $2 \mathrm{~d}$ sheet pattern design, $2 \mathrm{~d}$ pieces of $3 \mathrm{~d}$ display, and other functions, in the design of system development, the need to combine with large $3 \mathrm{~d}$ graphics library and powerful graphics functions OpcnGL technology and has strong data visualization and image processing, the equation of computing of MATLAB, through the body's $3 \mathrm{~d}$ grid model templates to build warp knitted seamless garment two-dimensional prototype pieces, eventually achieve the design of clothing design and virtual display in the computer.

Second, on the system architecture, it is composed of four layers structure, first of all, the first 
layer is the base class library, its phase 2 is geometric algorithm, $3 \mathrm{~d}$ model geometry processing, 3 $\mathrm{d}$ modeling, $3 \mathrm{~d}$ model rendering and display system. The third layer structure made by $2 \mathrm{~d}$ pattern design, the human body and garment processing, the last layer structure is a three-dimensional virtual fashion show, to realize the function of the last layer structure, must want to have the first three layer structure as the foundation, therefore, the system architecture has strong flexibility, low coupling characteristics.

Third, on the system function realization, mainly through concrete in the computer programming $\mathrm{c}++$ and Matlab code to implement some system functions, such as: the scene management, symbolic object, the geometric model, virtual camera, lighting, rendering, the human body and garment processing, etc.

Fourth, the system interface, through drawing tool to design the $2 \mathrm{~d}$ sheet, through the system display $3 \mathrm{~d}$ virtual clothing, again after the human body model import good pieces on the model displays a design, through the mouse to move, zoom, and other functions, the model can make the garment all-round display on the system.

\section{Conclusion}

In the era of the rapid development of science and technology, how to use computer to realize industry upgrading and development is to measure the production technical level and the national scientific and technological progress and industrial modernization. In the computer aided design of warp knitted seamless garment, its function is increasingly perfect, now can use CAD technology to complete the process layout, well block, discharge, etc., but still has great difficulty in clothing design, by reason of apparel orders, is various, product refinement of professional, made on the design of warp knitting seamless garment CAD still needs further study, so as to improve its function and improve the process efficiency.

\section{Reference}

[1] nan-nan zhang; Jiang gaoming. Wu zhiming. Warp knitted seamless jacket weaving process design [J] Shanghai textile science and technology, 2011 (3) : 36 to 38.

[2] Dong Zhijia; Jiang gaoming. Wu zhiming; Hong-lian cong. Ai-jun zhang. Warp knitting bottomless seamless fabric forming principle and the computer aided design [J] textile journal, 2015 (02) : 33-35.

[3] aqua regia. Ma y its; Close the will; Longhai as. High combed cotton warp knitted seamless garment development [J] knitting industry, 2014 (03) : 61-72. 